\title{
Simulating Vibronic Spectra without Born-Oppenheimer Surfaces
}

\author{
Kevin Lively, Guillermo Albareda, Shunsuke A. Sato, Aaron Kelly,* and Angel Rubio*
}

Cite This: J. Phys. Chem. Lett. 2021, 12, 3074-3081

Read Online

ABSTRACT: We show how linear vibronic spectra in molecular systems can be simulated efficiently using first-principles approaches without relying on the explicit use of multiple Born-Oppenheimer potential energy surfaces. We demonstrate and analyze the performance of mean-field and beyond-mean-field dynamics techniques for the $\mathrm{H}_{2}$ molecule in one dimension, in the later case capturing the vibronic structure quite accurately, including quantum FranckCondon effects. In a practical application of this methodology we simulate the absorption spectrum of benzene in full dimensionality using time-dependent density functional theory at the multitrajectory Ehrenfest level, finding good qualitative agreement with experiment and significant spectral reweighting compared to commonly used single-trajectory Ehrenfest dynamics. These results form the foundation for nonlinear spectral calculations and show promise for future application in capturing phenomena associated with vibronic coupling in more complex molecular and potentially condensed phase systems.

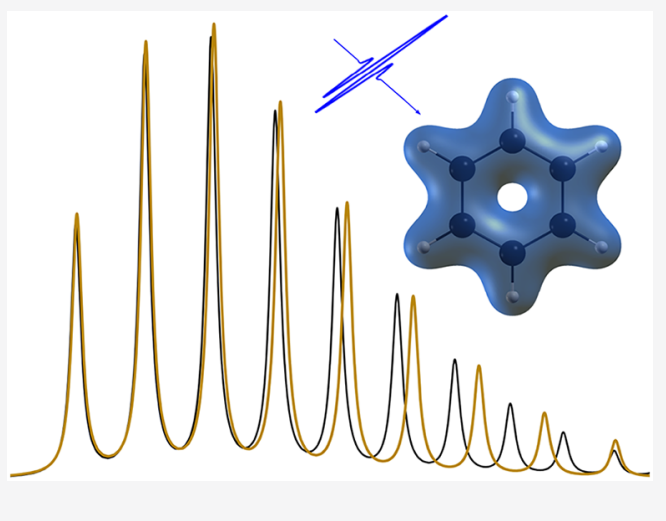

imulating vibronic effects from first-principles calculations $\checkmark$ is one of the central goals in theoretical spectroscopy that has implications in chemistry, physics, and materials science. The involvement of nuclear vibrational quantum states during electronic transitions plays a decisive role in determining the spectral features associated with these processes. This has been well-established by the utility of the Franck-Condon principle, for example, which represents an early paradigm for the role of nuclear quantum effects in electronically nonadiabatic processes. Describing this interplay between the electronic and vibrational degrees of freedom requires a quantum mechanical description that is both accurate and scalable to relatively large system sizes. One popular method to calculate vibronic spectra is to take a sum-over-states approach, where matrix elements of the transition operators between the states involved in generating the desired spectral signal are constructed. In this approach the states of interest can be represented using the Born-Oppenheimer (BO) basis; one must already have some a priori knowledge of the $\mathrm{BO}$ states that are involved, along with the associated potential energy surfaces and nonadiabatic couplings.

An alternative strategy to summing over states in the $\mathrm{BO}$ basis is to take a coordinate space perspective and construct the response function for the system of interest from direct time-propagation of the system in that picture. ${ }^{1,2}$ This invariably requires some level of approximation in the representation dynamics of the electronic and nuclear degrees of freedom, with different consequences for their coupling depending on the method chosen. The mixed quantumclassical Ehrenfest approach is a practical approximation to the fully quantum mechanical dynamics of the system, and despite its approximate dynamics, provides a formally exact representation of the quantum equilibrium structure of the correlated electronic and vibrational degrees of freedom via a multitrajectory Ehrenfest (MTEF) simulation through the use of the Wigner representation. ${ }^{3-5}$ In this case, the Wigner transform maps the vibrational quantum states onto phase space distributions of continuous position and momentum coordinates which can be sampled by an appropriate Monte Carlo procedure to capture the quantum equilibrium structure of the problem. The limitations of the Ehrenfest approach and other independent trajectory semiclassical methods are wellknown, ${ }^{6-10}$ and while there have been many attempts to ameliorate these shortcomings, with some exceptions, ${ }^{11,12}$ most rely on the $\mathrm{BO}$ framework in their implementation. ${ }^{13-17}$ In this work we take a different approach to go beyond meanfield theory based on the recently introduced interacting conditional wave function (ICWF) formalism, which is able to capture correlated electronic and nuclear dynamics. ${ }^{18-21}$ We apply MTEF and ICWF dynamics to an exactly solvable onedimensional $\mathrm{H}_{2}$ model and show that these methods are able to recover electron-nuclear correlations in linear vibronic spectra without the need to calculate multiple $B O$ surfaces. In addition, we show that the MTEF method can be easily extended to $a b$ initio nonadiabatic molecular dynamics

Received: January 8, 2021

Accepted: March 15, 2021

Published: March 22, 2021 
simulations by calculating the vibronic spectra for benzene, where we find good agreement with experimental results.

The linear spectrum of a system is given by the Fourier transform of time correlation function (TCF) $C_{A B}(t)=\langle[\hat{A}(t)$, $\hat{B}]\rangle$ of the transition dipole operator, $\hat{\mu}, C_{\mu \mu}(t)=\langle\hat{\mu}(t) \hat{\mu}(0)\rangle^{1,22}$ (unless otherwise stated all expressions are in atomic units):

$$
\begin{aligned}
I(\omega) & =\frac{4 \pi \omega}{3 c} \int_{-\infty}^{\infty} \mathrm{d} t \mathrm{e}^{\mathrm{i} \omega t}\langle[\hat{\mu}(t), \hat{\mu}]\rangle \\
& =\frac{8 \pi \omega}{3 c} \Re \int_{0}^{\infty} \mathrm{d} t \mathrm{e}^{\mathrm{i} \omega t} \operatorname{Tr}\left(\hat{\mu}(t) \hat{\mathrm{eq}}_{\mathrm{eq}} \hat{\mu}(t=0)\right)
\end{aligned}
$$

where the trace occurs over nuclear and electronic degrees of freedom; $\hat{\rho}_{\text {eq }}$ is the equilibrium density matrix for the coupled system, and we evolve $\hat{\mu}(t)$ in the Hilbert representation. Traditionally, vibronic spectra are explained by invoking the Franck-Condon approximation in the $\mathrm{BO}$ picture, where the electronic system is instantly excited, thus promoting the unperturbed ground-state nuclear system to a different electronic surface. If one has access to the electronic states involved in a particular spectral range then the contributions to the spectrum due to each electronic transition can be identified by resolving the transition dipole operator in the basis of the electronic states of interest, and the vibronic side peaks of that transition can be calculated by propagating the initial state's nuclear subsystem under the effect of the nonequilibrium electronic occupation. When it is feasible to resolve the nuclear wave function dynamics, this can be one of the most accurate methods of calculating molecular vibronic spectra. ${ }^{23,24}$

Although resolving eq 1 in the $\mathrm{BO}$ framework is a powerful analysis tool, it is computationally impractical for systems with many nuclear degrees of freedom, particularly when one desires spectra over multiple surfaces. One can bypass this computational bottleneck by representing the system in a real space basis and using the " $\delta$-kick" method, ${ }^{25}$ which captures electronic transitions to all dipole-transition allowed states (resolved on the grid) within a single calculation by utilizing the dipole response to a perturbative, but impulsive external field $\hat{H}_{\text {field }}=E(t) \hat{\mu}$, with $E(t)=\kappa \delta(t)$ and $\kappa \ll 1$. Using firstorder perturbation theory, the dipole response $\langle\Delta \mu(t)\rangle=$ $\langle\mu(t)\rangle-\langle\mu(0)\rangle$ can be written in powers of the field ${ }^{1,2}$

$$
\langle\Delta \mu(t)\rangle=i \operatorname{Tr}\left(\left[\hat{\mu}^{I}(t), \hat{\mu}^{I}(0)\right] \hat{\rho}_{\mathrm{eq}}\right) \kappa+O\left(\kappa^{2}\right)
$$

where $\hat{\mu}^{I}(t)$ is evolved in the interaction representation. Hence, the linear response spectra may also be obtained via the relation

$$
C_{\mu \mu}(t)=\frac{-i}{\kappa}\langle\Delta \mu(t)\rangle
$$

provided the strength of the perturbing field, $\kappa$, is sufficiently small. This $\delta$-kick approach requires only the initial state of the full system as input, followed by time propagation for a sufficient duration so as to obtain the desired energy resolution. Importantly, this technique can also serve as a foundation for calculating nonlinear optical response spectra. ${ }^{26}$

While the methods described above are formally equivalent, differences between the calculated spectra can arise when approximations are made. Here we briefly describe two methods for performing coupled electron nuclear dynamics simulations: the quantum-classical mean-field MTEF method and the ICWF formalism, which was designed to go beyond the mean-field limit.
A typical approach to Ehrenfest theory is to assume a separable electronic-nuclear wave function ansatz, take the classical limit of the nuclear portion, and initialize the nuclei at the equilibrium position with zero nuclear momentum. ${ }^{27,28}$ This single-trajectory Ehrenfest (STEF) method is often employed when a mixed quantum-classical method is needed to couple electronic and nuclear dynamics, ${ }^{29}$ in some cases providing a stark difference in electronic dynamics compared to fixed nuclei. ${ }^{30,31}$ Although attempts at capturing quantized vibrational effects in STEF with the $\delta$-kick method have been made, $^{32}$ they can contain unphysical spectral features (see the Supporting Information) which make them unsuitable for application to nonlinear spectra

An alternative route to Ehrenfest is also possible in the density matrix picture and proceeds via the quantum-classical Liouville equation. $^{33}$ The major difference is that this representation results in a multitrajectory Ehrenfest picture of the dynamics, where the initial quantum statistics of the correlated system can, in principle, be captured exactly. Here, we outline the evolution equations, and we offer more details in the Supporting Information. The time evolution of the reduced electronic density is

$$
\frac{\mathrm{d}}{\mathrm{d} t} \hat{\rho}_{\mathrm{e}}(t)=-i\left[\hat{H}_{\mathrm{e}, \mathrm{W}}^{\mathrm{Eff}}(\mathbf{X}(t)), \hat{\rho}_{\mathrm{e}}(t)\right]
$$

where the subscript $\mathrm{W}$ refers to the partial Wigner transform over the nuclei; $\mathbf{X}=(\mathbf{R}, \mathbf{P})$ is a collective variable for the nuclear position $\mathbf{R}$ and momentum $\mathbf{P}$, and the effective electronic mean-field Hamiltonian is $\hat{H}_{\mathrm{e}, \mathrm{W}}^{\mathrm{Eff}}(\mathbf{X}(t))=\hat{H}_{\mathrm{e}}+$ $\hat{H}_{\text {en, }}(\mathbf{X}(t))$, where $\hat{H}_{\mathrm{e}}$ refers to the electronic portion of the Hamiltonian and $\hat{H}_{\text {en }}$ to the electron nuclear coupling. The nuclear dynamics is represented as an ensemble of $N$ independent Wigner phase-space trajectories, $\rho_{\mathrm{n}, \mathrm{W}}(\mathbf{X}, t)=1 /$ $N \sum_{i}^{N} \delta\left(\mathbf{X}_{i}-\mathbf{X}_{i}(t)\right)$, that evolve according to Hamilton's equations of motion generated from the effective nuclear mean-field Hamiltonian

$$
\begin{aligned}
\frac{\partial \mathbf{R}_{i}}{\partial t} & =\frac{\partial H_{\mathrm{n}, \mathrm{W}}^{\mathrm{Eff}}}{\partial \mathbf{P}_{i}}, \frac{\partial \mathbf{P}_{i}}{\partial t}=-\frac{\partial H_{\mathrm{n}, \mathrm{W}}^{\mathrm{Eff}}}{\partial \mathbf{R}_{i}} \\
H_{\mathrm{n}, \mathrm{W}}^{\mathrm{Eff}} & =H_{\mathrm{n}, \mathrm{W}}\left(\mathbf{R}_{i}(t)\right)+\operatorname{Tr}_{\mathrm{e}}\left(\hat{\rho}_{\mathrm{e}}(t) \hat{H}_{\mathrm{en}, \mathrm{W}}\left(\mathbf{X}_{i}(t)\right)\right)
\end{aligned}
$$

The average value of any observable, $\langle O(t)\rangle$, can then be written as

$$
\langle O(t)\rangle=\operatorname{Tr}_{\mathrm{e}} \int \mathrm{d} \mathbf{X} \hat{O}_{\mathrm{W}}(\mathbf{X}, t) \hat{\rho}_{\mathrm{W}}(\mathbf{X}, 0)
$$

which can be evaluated by sampling initial conditions from $\hat{\rho}_{\mathrm{W}}(\mathbf{X}, 0)$ and evolving the expectation value of the observable according to the above equations of motion. Using this dynamics method in conjunction with the $\mathrm{BO}$ basis representation to evaluate eqs 1 and 4-6 ultimately leads to the following equations of motion, with sums over BO states denoted by $a$ (see the Supporting Information for details) 


$$
\begin{aligned}
& \partial_{t} \rho_{\mathrm{e}}^{a a^{\prime}}=-i \rho_{\mathrm{e}}^{a a^{\prime}}(t)\left(\epsilon_{a}\left(\mathbf{R}_{i}(t)\right)-\epsilon_{a^{\prime}}\left(\mathbf{R}_{i}(t)\right)\right) \\
&+\sum_{a^{\prime \prime}} \frac{\mathbf{P}_{i}(t)}{M}\left(\rho_{\mathrm{e}}^{a a^{\prime \prime}}(t) d_{a^{\prime \prime} a^{\prime}}^{i}(t)-d_{a a^{\prime \prime}}^{i}(t) \rho_{\mathrm{e}}^{a^{\prime \prime} a^{\prime}}(t)\right) \\
& \partial_{t} \mathbf{R}_{i}(t)=\mathbf{P}_{i}(t) / M \\
& \partial_{t} \mathbf{P}_{i}(t)=\sum_{a}-\partial_{\mathbf{R}} \epsilon_{a}\left(\mathbf{R}_{i}(t)\right) \rho_{\mathrm{e}}^{a a}(t) \\
&+\sum_{a a^{\prime}} \mathfrak{R}\left[\left(\epsilon_{a}\left(\mathbf{R}_{i}(t)\right) d_{a a^{\prime}}^{i}(t)-\epsilon_{a^{\prime}}\left(\mathbf{R}_{i}(t)\right) d_{a^{\prime} a}^{i}(t)\right) \rho_{\mathrm{e}}^{a^{\prime} a}(t)\right] \\
& \partial_{t} \mu_{W}^{a a^{\prime}}\left(\mathbf{R}_{i}(t)\right)=i \mu_{W}^{a a^{\prime}}\left(\mathbf{R}_{i}(t)\right)\left(\epsilon_{a}\left(\mathbf{R}_{i}(t)\right)-\epsilon_{a^{\prime}}\left(\mathbf{R}_{i}(t)\right)\right)
\end{aligned}
$$

where $\epsilon_{a}(\mathbf{R})$ are the BO surfaces and $d_{a a^{\prime}}$ are the nonadiabatic coupling vectors (NACVs) between states $a$ and $a^{\prime}$.

In contrast to the previous expression, utilizing MTEF in the real space $\delta$-kick approach requires initializing the electronic wave function as the $\mathrm{BO}$ eigenstate for each initially sampled nuclear geometry. The $\delta$-kick is applied and the electronic wave function is propagated using the time-dependent Schrödinger equation equivalent to eq 4 alongside the nuclei according to eq 5. Calculating the spectrum via MTEF dynamics in the $\mathrm{BO}$ picture is from here on referred to as MTEF-BO, and calculating it via the $\delta$-kick method is referred to as MTEF-kick.

Moving beyond semiclassical dynamics, the formally exact CWF method and its practical ICWF implementation are recently developed methods which have shown to be able to capture nonequilibrium correlated nuclear-nuclear and electron-nuclear phenomena beyond the mean-field limit. ${ }^{18-21}$ This approach is based on taking single-particle slices (the CWFs) of the time-dependent wave function of the full system; approximating the equations of motion for these CWFs by the Hermitian components of the sliced Hamiltonian; and finally, in the ICWF extension, utilizing these electron-nuclear CWFs as a basis of Hartree products in a wave function ansatz.

Here we describe an implementation of this approach utilizing the static and time-dependent variational principles for the expansion coefficients in a static CWF basis. The basis is chosen via sampling electronic and nuclear positions $\left(\mathbf{r}^{\alpha}, \mathbf{R}^{\alpha}\right)$, $\alpha \in\left\{1, \cdots, N_{\mathrm{c}}\right\}$, where $\mathbf{r}$ and $\mathbf{R}$ are understood to be collective position variables, from initial guesses to the electronic and nuclear densities. These are used to construct the Hermitian limit of the CWF propagators ${ }^{18}$

$$
\begin{gathered}
h_{\mathrm{e}}^{\alpha}\left(\mathbf{r}_{i}\right)=-\frac{1}{2} \nabla_{\mathbf{r}_{i}}^{2}+\sum_{j \neq i}^{N_{e}} V_{e e}\left(\mathbf{r}_{i}, \mathbf{r}_{j}^{\alpha}\right)+\sum_{l}^{N_{\mathrm{n}}} V_{\mathrm{en}}\left(\mathbf{r}_{i}, \mathbf{R}_{l}^{\alpha}\right) \\
h_{\mathrm{n}}^{\alpha}\left(\mathbf{R}_{l}\right)=-\frac{1}{2 M_{l}} \nabla_{\mathbf{R}_{l}}^{2}+\sum_{j}^{N_{e}} V_{\mathrm{en}}\left(\mathbf{R}_{l}, \mathbf{r}_{j}^{\alpha}\right)+\sum_{m \neq l}^{N_{\mathrm{n}}} V_{\mathrm{nn}}\left(\mathbf{R}_{l}, \mathbf{R}_{m}^{\alpha}\right)
\end{gathered}
$$

for a system with $N_{\mathrm{e}}$ electrons and $N_{\mathrm{n}}$ nuclear degrees of freedom. Taking eigenstates of $h_{\mathrm{e}}^{\alpha}\left(\mathbf{r}_{i}\right)$ and $h_{\mathrm{n}}^{\alpha}\left(\mathbf{R}_{l}\right)$, denoted $\phi^{\alpha}\left(\mathbf{r}_{i}\right)$ and $\chi^{\alpha}\left(\mathbf{R}_{l}\right)$. respectively, as our (static) CWF basis we write the following single-index, multiconfigurational, mixedspecies, wave function ansatz:

$$
\Psi(\mathbf{r}, \mathbf{R}, t)=\sum_{\alpha}^{N_{c}} C_{\alpha}(t) \prod_{i}^{N_{e}} \phi^{\alpha}\left(\mathbf{r}_{i}\right) \prod_{l}^{N_{\mathrm{n}}} \chi^{\alpha}\left(\mathbf{R}_{l}\right)
$$

where we have taken a Hartree product of electronic and nuclear CWFs for each degree of freedom. While the Hartree product over electronic degrees of freedom and a single expansion index has been sufficient for accuracy in applications of ICWF so far, this ansatz can in principle be trivially extended to a multi-index expansion and to have Fermionic antisymmetry via inclusion of Slater determinants. We then utilize the Dirac-Frenkel variational procedure ${ }^{34-36}$ to develop equations of motion for $\vec{C}(t)$, which leads to the following standard evolution equation for the expansion coefficients of a nonorthogonal static basis:

$$
\frac{\mathrm{d}}{\mathrm{d} t} \vec{C}=-i \mathbf{S}^{-1} \mathbf{H} \vec{C}
$$

where

$$
\begin{aligned}
& \mathbf{S}_{\alpha \beta}=\prod_{i}^{N_{\mathrm{e}}} \int \mathrm{d} \mathbf{r}_{i}\left(\phi^{\alpha}\left(\mathbf{r}_{i}\right)\right)^{*} \phi^{\beta}\left(\mathbf{r}_{i}\right) \prod_{l}^{N_{\mathrm{n}}} \int \mathrm{d} \mathbf{R}_{l}\left(\chi^{\alpha}\left(\mathbf{R}_{l}\right)\right) * \chi^{\beta}\left(\mathbf{R}_{l}\right) \\
& \mathbf{H}_{\alpha \beta}=\prod_{i}^{N_{\mathrm{e}}} \prod_{l}^{N_{\mathrm{n}}} \int \mathrm{d} \mathbf{R}_{l} \mathrm{~d} \mathbf{r}_{i}\left(\phi^{\alpha}\left(\mathbf{r}_{i}\right) \chi^{\alpha}\left(\mathbf{R}_{l}\right)\right) * \hat{H}(\mathbf{r}, \mathbf{R}) \phi^{\beta}\left(\mathbf{r}_{i}\right) \chi^{\beta}\left(\mathbf{R}_{l}\right)
\end{aligned}
$$

for the full molecular Hamiltonian $\hat{H}$.

While the general form of this wave function ansatz is not unique, the mixed-species CWF basis treats the electronic and nuclear subsystems on an equal footing without relying on any adjustable parameters, using the Hermitian limit of the solutions to the conditionalized time-independent Schrödinger equations.

The ground-state wave function is obtained from this approach using imaginary time evolution, ${ }^{37,38}$ and the $\delta$-kick spectra (ICWF-kick) is calculated by applying the perturbative field to the CWFs at time zero and recalculating the $\mathbf{S}$ and $\mathbf{H}$ matrices, equivalent to propagating in the interaction representation. In practice, $\boldsymbol{S}$ may be nearly singular, but its inverse can be approximated by the Moore-Penrose pseudoinverse. ${ }^{39}$ This "closed-loop" of initial state preparation and time-propagation ensures that our ICWF approach is a fully self-consistent method that increases in accuracy with increasing $N_{\mathrm{c}}$ and requires no $\mathrm{BO}$ state information.

To investigate the performance of the MTEF and ICWF approaches to vibronic spectral lineshapes we studied the vibronic transitions in an exactly solvable one-dimensional model system for molecular hydrogen. ${ }^{40-42}$ The total Hamiltonian can be written in the center of mass frame in atomic units as

$$
\begin{gathered}
\hat{H}\left(r_{1}, r_{2}, R\right)=-\frac{\partial_{R}^{2}}{2 \mu_{\mathrm{n}}}-\sum_{i=1}^{2} \frac{\partial_{r_{i}}^{2}}{2 \mu_{\mathrm{e}}}+\frac{1}{\sqrt{\left(r_{1}-r_{2}\right)^{2}+1}}+\frac{1}{R} \\
-\sum_{i=1}^{2}\left(\frac{1}{\sqrt{\left(r_{i}+\frac{1}{2} R\right)^{2}+1}}+\frac{1}{\sqrt{\left(r_{i}-\frac{1}{2} R\right)^{2}+1}}\right)
\end{gathered}
$$

where $\mu_{\mathrm{n}}=m_{\mathrm{p}} / 2$ and $\mu_{\mathrm{e}}=2 m_{\mathrm{p}} /\left(2 m_{\mathrm{p}}+1\right)$ are the reduced nuclear and electronic masses; $R$ is the internuclear separation, and $r_{i}$ are the electronic positions. We take the proton mass to be $m_{\mathrm{p}}=1836$. The electronic and nuclear degrees of freedom were each resolved on grids for the numerically exact solution and ICWF-kick approaches, while the MTEF-kick electronic wave functions were time-evolved on the $\left(r_{1}, r_{2}\right)$ grid, and the 

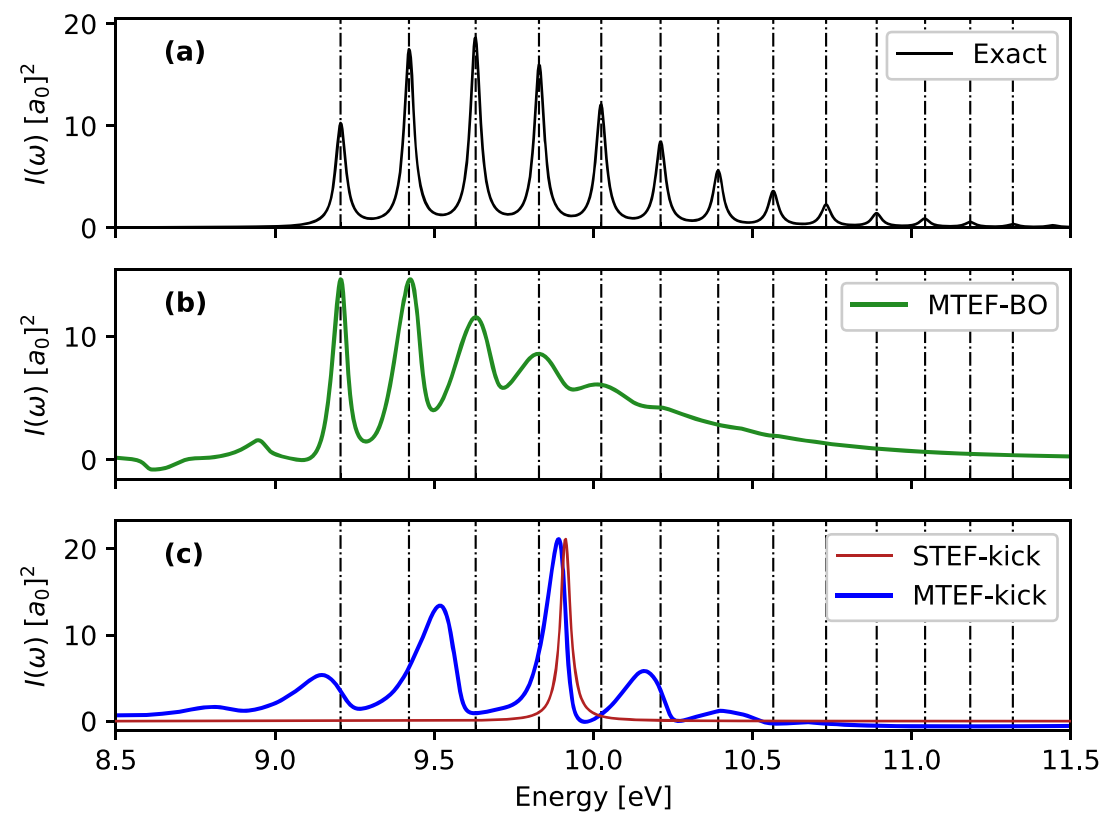

Figure 1. 1D $\mathrm{H}_{2}, S_{2} \leftarrow S_{0}$ spectra calculated via the MTEF-TCF, MTEF-kick, and STEF-kick approaches, with the exact peak placements overlaid as dashed vertical lines. Spectral cross sections are reported in square Bohr radii $a_{0}^{2}$. For clarity the STEF-kick spectrum has been multiplied by a factor of 0.175 to match the scale of the MTEF-kick results.
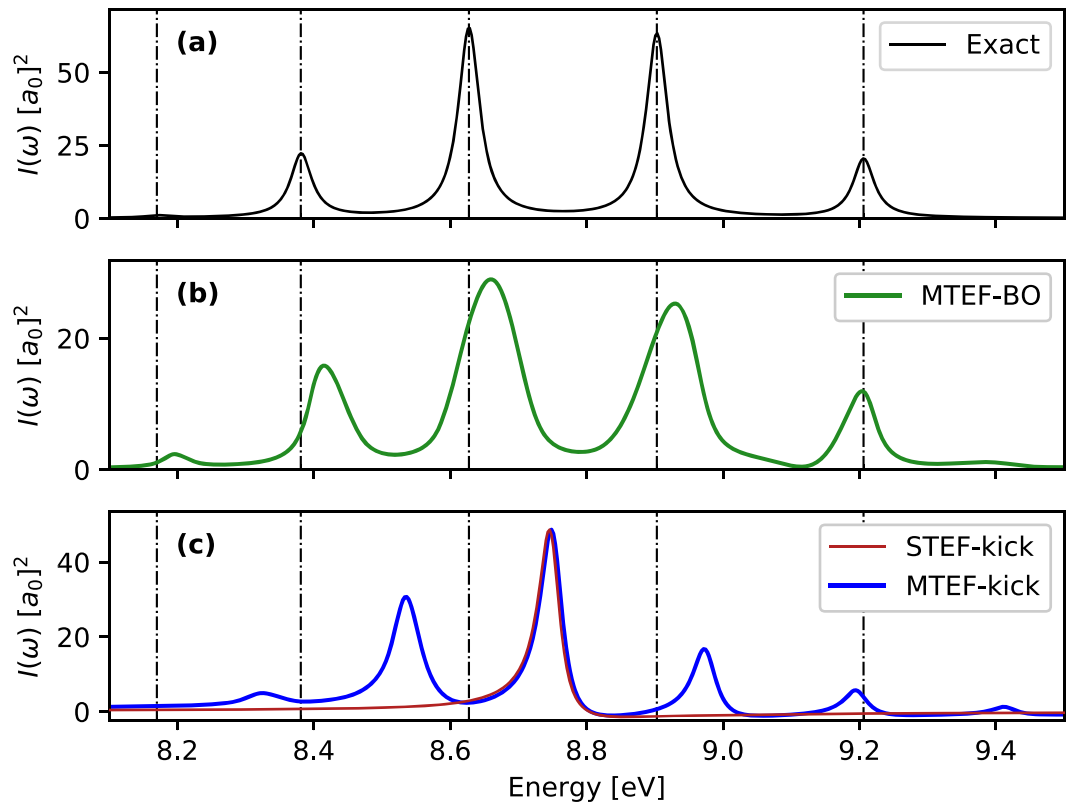

Figure 2. $S_{0} \leftarrow S_{2}$ spectra compared between the MTEF-TCF, MTEF-kick, and STEF-kick approaches, with exact peak placement overlaid as dashed vertical lines. MTEF nuclear initial conditions are sampled from the lowest-lying vibrational state on $S_{2}$. The sign of all spectra here is inverted for ease of comparison to other figures, and for legibility the STEF-kick spectrum was multiplied by a factor of 0.4 to match the MTEFkick spectra maximum.

MTEF-BO information was calculated by solving the electronic subsystem across the nuclear grid; see Computational Methods for more details. A kick strength of $\kappa=$ $10^{-4} a_{0}{ }^{-1}$. was sufficient to generate the kick spectra within the linear response regime and, unless otherwise stated, a total propagation time of $10000 \mathrm{au} \approx 242$ fs was used to generate the spectra.

In Figure 1 we show mean-field spectra calculated both with (MTEF-BO) and without (MTEF-kick) the use of multiple BO surfaces for the absorption from $S_{0}$ to $S_{2}$ in comparison with the numerically exact results. We see that in the $\mathrm{BO}$ picture the MTEF method recovers the vibronic absorption peak placement quite accurately for the first five peaks, with a broadening occurring for the higher-energy peaks that leads to a loss of structure. This broadening of the spectral signal is due to the well-known fact that the MTEF dynamics does not preserve the correct quantum statistics and thus cannot fully capture the electron-nuclear correlation in the problem (see the Supporting Information for a detailed discussion of this issue). The prepeak features in Figure $1 \mathrm{~b}$ are also unphysical artifacts of MTEF. The MTEF-BO spectra were converged to within graphical accuracy using $N=50000$ trajectories, 

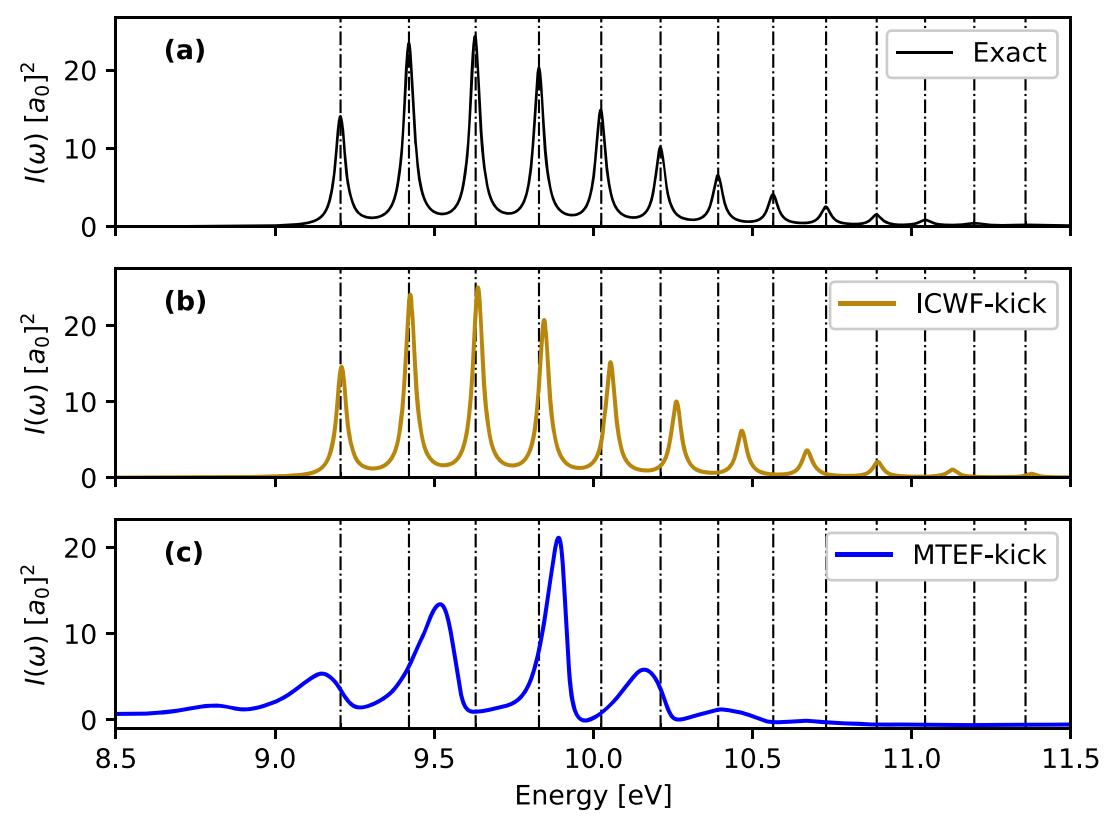

Figure 3. $S_{2} \leftarrow S_{0}$ spectra of the ICWF-kick and MTEF-kick methods, with the exact peak placement overlaid as dashed lines.

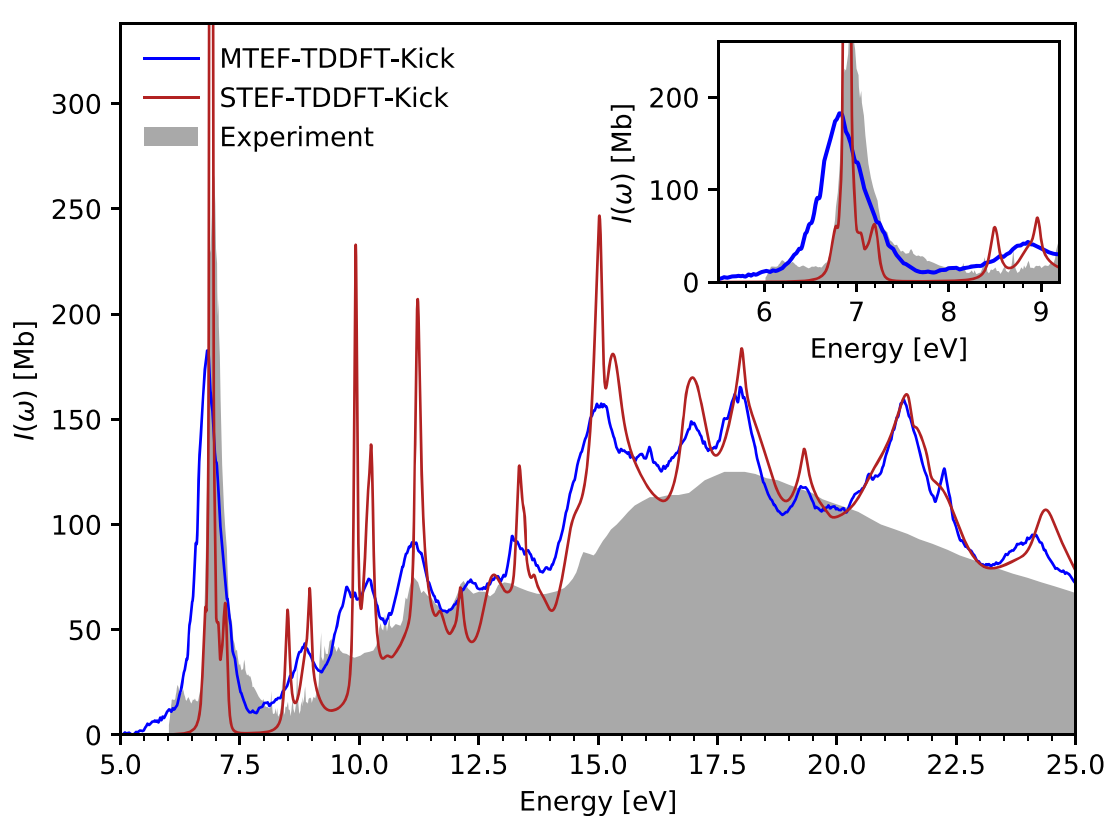

Figure 4. Experimental vibronic spectra for the lowest-lying optical transitions of benzene ${ }^{46}$ compared to the MTEF and STEF kick spectra calculated with TDDFT.

although an ensemble size of approximately $N=500-1000$ also yields reasonable results.

Focusing on the MTEF-kick results in Figure 1c, we see that this approach recovers vibronic side peak structures again without any BO surface information, albeit with inaccurate spacing, while STEF-kick captures only the vertical electronic transition from the minimum of the $S_{0}$ surface. The average peak spacing in the MTEF-kick spectra is approximately 0.32 $\mathrm{eV}$; this corresponds remarkably well with the natural frequency of the harmonic approximation to the ground-state surface expanded around the equilibrium geometry, which is also $0.32 \mathrm{eV}$ in this case. This result is unsurprising as the electronic kick induces a very small population transfer to the upper surface proportional to the square of the kick strength, which results in the mean forces on the nuclei in MTEF-kick essentially corresponding to those of the initial state.

The influence of the initial state on the MTEF-kick spectra is further demonstrated by analyzing the emission spectra in Figure 2. The initial state here was chosen by hand as the lowest-lying nuclear state on the $S_{2}$ surface. Once again we see that MTEF-BO recovers the peak placement quite well, while the MTEF-kick data has a less accurate vibronic spacing. Fitting the MTEF-kick peaks, we find an excellent correspondence between mean spacing of the five lowestenergy MTEF peaks and the excited surface natural frequency of $0.21 \mathrm{eV}$. Although the nuclear dynamics within MTEF-kick are primarily governed by the properties of the initial electronic state, the electron-nuclear coupling modulates the electronic linear response in a nontrivial manner, fundamen- 
tally changing the system response calculation compared to simply averaging the electronic transition properties over the equilibrium nuclear configuration, as is done in the nuclear ensemble approach ${ }^{43}$ (see the Supporting Information).

For ICWF-kick, we found that $N_{c}=4096$ and mixing the three lowest-energy CWF eigenstates in roughly equal proportion was sufficient to obtain quite accurate results. In Figure 3 we demonstrate that the ICWF ansatz used in a variational context achieves a much more accurate vibronic spacing than the MTEF-kick approach, without the failing of peak broadening or unphysical spectral negativity apparent in the MTEF-BO results. The accuracy of these results underscores that the ICWF ansatz is a robust framework to capture the electronic and vibronic quantum dynamics, being accurate for not only the electron-nuclear correlation inherent to vibronic spectra but also the electronic subsystem itself, which in the MTEF results was solved exactly either on a grid or using explicit $\mathrm{BO}$ state information. The deviation from the exact results does grow with increasing energy, although this is ameliorated with increasing $N_{c}$ and can in principle be eliminated at large enough values of $N_{c}$ (see the Supporting Information).

Finally we demonstrate the application of MTEF-kick to real $3 \mathrm{D}$ molecular systems using the $a b$ initio Octopus ${ }^{44}$ real-space time-dependent density functional theory (TDDFT) ${ }^{45}$ package to calculate the linear vibronic MTEF-kick spectra of benzene. The initial conditions for the nuclear subsystem were obtained by calculating the normal-mode frequencies and dynamical matrix of the molecule and sampling Wigner transforms of the ground-state wave functions in the harmonic approximation (see the Supporting Information for more details). The adiabatic-LDA functional was used, along with normconserving Troullier-Martins pseudopotentials, and the trajectories were evolved for $201 \frac{\hbar}{\mathrm{eV}} \approx 132$ fs with a time step of $\Delta t=0.0015 \frac{\hbar}{\mathrm{eV}} \approx 1$ as. A kick strength of $\kappa=5 \times 10^{-3}$ $\AA^{-1}$ was used to generate the kick spectra within the linear response regime in this case, and the graphical convergence of the MTEF results was found to be achieved with $N=500$ trajectories.

In Figure 4, we compare the MTEF-TDDFT-kick results, its STEF-TDDFT-kick counterpart, and gas-phase experimental data. ${ }^{46}$ There is remarkably good agreement across the wide energy range available from experiment, before molecular dissociation pathways become available around $13.8 \mathrm{eV}$. Again, this full linear absorption spectrum is obtained without resorting to the calculation of individual transitions between states as would be required in a BO-state-based calculation. Principally, there is a significant spectral reweighting between STEF and MTEF below $17.5 \mathrm{eV}$, above which the electronic density of states is so high as to obscure the difference between the two methods. In the inset of Figure 4, in the $7 \mathrm{eV}$ region corresponding to the energy range of the doubly degenerate, dipole-allowed ${ }^{1} E_{1 u} \leftarrow{ }^{1} A_{1 g}, \quad \pi^{*} \leftarrow \pi$ transition, ${ }^{31,47,48}$ the STEF spectral weight is distributed across a much wider energy range in the MTEF signal, encompassing the experimental bands from 6 to $8 \mathrm{eV}$. The two STEF peaks at 8.5 and $8.95 \mathrm{eV}$ are also spread across the $8-9 \mathrm{eV}$ range. It is reasonable to expect that the broadening of the MTEF signal relative to the experimental signal is due to the effects discussed above that arise because of the mean-field treatment. In the Supporting Information we also compare these results to the broadening from the nuclear ensemble average calculation of the spectrum and find good agreement, given the high density of electronic states and many nuclear degrees of freedom in this system. By comparison with the standard STEF dynamics results used in large $a b$ initio simulations, we see that utilizing multiple trajectories with equilibrium quantum nuclear statistics fundamentally changes the properties of the spectrum.

We have demonstrated that semiclassical MTEF simulations can capture vibronic structure with the correct spectral sign in the region of the transition. Moreover, we have shown how this can be achieved without using multiple BO surfaces via the $\delta$ kick method and that the vibronic spacing calculated with the MTEF-kick approach matches the profile of the initial state. We have shown that utilizing a dynamics method that can accurately capture the correlated electron-nuclear dynamics, such as the ICWF method, in tandem with the $\delta$-kick approach allows one to accurately recover the vibronic spectra. Finally, we demonstrated that MTEF-kick is easily applied to ab initio molecular systems by simulating the vibronic spectra of benzene and finding good agreement to experimental results.

These linear response results establish a solid basis for further investigations into nonlinear response of field driven molecular systems utilizing the practical and efficient MTEF and ICWF techniques along with $a b$ initio electronic structure methods. Work in preparation by the present authors also explores the utility of ICWF with electron-electron and electron-nuclear correlated systems and explores the response of these systems under nonperturbative electric fields. Furthermore, we expect that MTEF-kick will improve in accuracy for periodic systems, as changes in the electronic configuration are often to likely produce smaller changes in the nuclear forces than in molecular hydrogen. This makes this method interesting to pursue in periodic systems in particular, where there is a dearth of theoretical frameworks for $a b$ initio, nonpertubrative electron-nuclear coupling. ${ }^{49}$ Work in this direction is in progress, as is the implementation of the ICWF method within an $a b$ initio framework for molecular and periodic systems.

\section{COMPUTATIONAL METHODS}

In the $1 \mathrm{D} \mathrm{H}_{2}$ model, the electronic coordinates are each resolved on a $65 a_{0}$ wide interval with spacing of $0.6 a_{0}$, while the nuclear grid extends to $R_{\max }=6.3125 a_{0}$ with $0.0625 a_{0}$ spacing. Quadratic complex absorbing potentials were also added to the Hamiltonian to prevent reflection from the simulation box edge (see the Supporting Information). To generate the exact results we evolved the full wave function under the $\delta$-kick on the three-dimensional electron-nuclear grid, while for MTEF-kick, the electronic subsystem's Schrödinger equation, dependent on $\mathbf{R}_{i}(t)$, was solved exactly on the two-dimensional electronic grid for each trajectory. All wave functions were time-propagated using a fourth-order Runge-Kutta integration scheme with a time-step size of $\Delta t=$ 0.05 au. For the MTEF trajectories, the nuclear degree of freedom was propagated via a velocity Verlet type scheme with the same time-step size. ${ }^{50}$ An exponential damping mask function $\exp (-\gamma t)$ was applied to all time-dependent signals in the Fourier transform, and the damping factor was set to damp the signal to $0.1 \%$ of its strength at the final time.

For the $1 \mathrm{D} \mathrm{H}_{2}$ MTEF-BO results, the potential energy surfaces $\epsilon_{a}(R)$ and $\mu_{\mathrm{W}}^{a a \prime}(R)$ were calculated on a nuclear grid with $\Delta R=0.02 a_{0}$ up to $R_{\max }=8 a_{0}$, fit to a cubic spline function, and interpolated every $0.01 \Delta R$. The NACV between 
$S_{0}$ and $S_{2}$ in this model is numerically zero. These quantities were resolved for the first allowed dipole transition, between the ground state $\left(S_{0}\right)$ and the second excited state $\left(S_{2}\right)$, and the results were found to be well converged within about $5 \times 10^{4}$ trajectories.

For the MTEF-TDDFT-kick simulations we used a real space grid formed from overlapping spheres of radius $8 \AA$ centered on the initial positions of the nuclei, with an isotropic grid spacing of $0.16 \AA$, which was found to be sufficient to converge the energies of the lowest-lying absorption lines. The reported results were calculated on a hyperthreaded $16 \mathrm{CPU}$ core Xeon E5-2698 v3 requiring approximately 880 core hours per trajectory. Being composed of independent trajectories the cost of the MTEF method over the STEF simulation scales linearly with the number of trajectories, requiring approximately 440000 core hours for graphical convergence at 500 trajectories.

\section{ASSOCIATED CONTENT}

\section{SI Supporting Information}

The Supporting Information is available free of charge at https://pubs.acs.org/doi/10.1021/acs.jpclett.1c00073.

Details on MTEF, STEF, and ICWF as referenced in the main text (PDF)

\section{AUTHOR INFORMATION}

\section{Corresponding Authors}

Aaron Kelly - Max Planck Institute for the Structure and Dynamics of Matter and Center for Free-Electron Laser Science, 22761 Hamburg, Germany; Department of Chemistry, Dalhousie University, Halifax B3H 4R2, Canada; Email: aaron.kelly@mpsd.mpg.de

Angel Rubio - Max Planck Institute for the Structure and Dynamics of Matter and Center for Free-Electron Laser Science, 22761 Hamburg, Germany; Nano-Bio Spectroscopy Group and ETSF, Universidad del País Vasco, 20018 San Sebastían, Spain; Center for Computational Quantum Physics (CCQ), Flatiron Institute, New York 10010, United States; orcid.org/0000-0003-2060-3151;

Email: angel.rubio@mpsd.mpg.de

\section{Authors}

Kevin Lively - Max Planck Institute for the Structure and Dynamics of Matter and Center for Free-Electron Laser Science, 22761 Hamburg, Germany; (1) orcid.org/00000003-2098-1494

Guillermo Albareda - Max Planck Institute for the Structure and Dynamics of Matter and Center for Free-Electron Laser Science, 22761 Hamburg, Germany; Institute of Theoretical and Computational Chemistry, University of Barcelona, 08028 Barcelona, Spain; Nano-Bio Spectroscopy Group and ETSF, Universidad del País Vasco, 20018 San Sebastían, Spain; orcid.org/0000-0002-2181-7023

Shunsuke A. Sato - Max Planck Institute for the Structure and Dynamics of Matter and Center for Free-Electron Laser Science, 22761 Hamburg, Germany; Center for Computational Sciences, University of Tsukuba, Tsukuba 305-8577, Japan

Complete contact information is available at: https://pubs.acs.org/10.1021/acs.jpclett.1c00073

\section{Notes}

The authors declare no competing financial interest.

\section{ACKNOWLEDGMENTS}

This work was supported by the European Research Council (ERC-2015-AdG694097), the Cluster of Excellence Advanced Imaging of Matter (AIM), JSPS KAKENHI Grant Number 20K14382, Grupos Consolidados (IT1249-19), and SFB925. The Flatiron Institute is a division of the Simons Foundation.

\section{REFERENCES}

(1) May, V.; Kühn, O. Charge and Energy Transfer Dynamics in Molecular Systems, 3rd ed.; 2011.

(2) Ullrich, C. A. Time-Dependent Density-Functional Theory: Concepts and Applications; Oxford Graduate Texts; 2011.

(3) Wigner, E. On the quantum correction for thermodynamic equilibrium. Phys. Rev. 1932, 40, 749-759.

(4) Case, W. B. Wigner functions and Weyl transforms for pedestrians. Am. J. Phys. 2008, 76, 937-946.

(5) Grunwald, R.; Kelly, A.; Kapral, R. Quantum Dynamics in Almost Classical Environments. In Energy Transfer Dynamics in Biomaterial Systems, 2009.

(6) Jasper, A. W.; Zhu, C.; Nangia, S.; Truhlar, D. G. Introductory lecture: Nonadiabatic effects in chemical dynamics. Faraday Discuss. 2004, 127, 1-22.

(7) Karsten, S.; Ivanov, S. D.; Bokarev, S. I.; Kühn, O. Quasi-classical approaches to vibronic spectra revisited. J. Chem. Phys. 2018, 102337.

(8) Tully, J. C. Mixed quantum-classical dynamics. Faraday Discussions 1998, 110, 407.

(9) Kapral, R. Progress in the theory of mixed quantum-classical dynamics. Annu. Rev. Phys. Chem. 2006, 57, 129-157.

(10) Lee, M. K.; Huo, P.; Coker, D. F. Semiclassical Path Integral Dynamics: Photosynthetic Energy Transfer With Realistic Environment Interactions. Annu. Rev. Phys. Chem. 2016, 67, 639-668.

(11) Agostini, F.; Min, S. K.; Abedi, A.; Gross, E. K. U. Quantumclassical nonadiabatic dynamics: coupled- vs independent-trajectory methods. J. Chem. Theory Comput. 2016, 12, 2127-2143.

(12) Talotta, F.; Agostini, F.; Ciccotti, G. Quantum trajectories for the dynamics in the exact factorization framework: A proof-ofprinciple test. J. Phys. Chem. A 2020, 124, 6764-6777.

(13) Tully, J. C. Molecular dynamics with electronic transitions. J. Chem. Phys. 1990, 93, 1061-1071.

(14) Donoso, A.; Martens, C. C. Simulation of coherent nonadiabatic dynamics using classical trajectories. J. Phys. Chem. A 1998, 102, 4291-4300.

(15) Shalashilin, D. V. Multiconfigurational Ehrenfest approach to quantum coherent dynamics in large molecular systems. Faraday Discuss. 2011, 153, 105-116.

(16) Mignolet, B.; Curchod, B. F. A walk through the approximations of ab initio multiple spawning. J. Chem. Phys. 2018, $148,134110$.

(17) Nijjar, P.; Jankowska, J.; Prezhdo, O. V. Ehrenfest and classical path dynamics with decoherence and detailed balance. J. Chem. Phys. 2019, 150, 204124.

(18) Albareda, G.; Appel, H.; Franco, I.; Abedi, A.; Rubio, A. Correlated electron-nuclear dynamics with conditional wave functions. Phys. Rev. Lett. 2014, 113, 083003.

(19) Albareda, G.; Bofill, J. M.; Tavernelli, I.; Huarte-Larranaga, F.; Illas, F.; Rubio, A. Conditional Born-Oppenheimer dynamics: Quantum dynamics simulations for the model porphine. J. Phys. Chem. Lett. 2015, 6, 1529.

(20) Albareda, G.; Abedi, A.; Tavernelli, I.; Rubio, A. Universal steps in quantum dynamics with time-dependent potential-energy surfaces: Beyond the Born-Oppenheimer picture. Phys. Rev. A: At., Mol., Opt. Phys. 2016, 94, 062511.

(21) Albareda, G.; Kelly, A.; Rubio, A. Nonadiabatic quantum dynamics without potential energy surfaces. Physical Review Materials 2019, 3, 023803. 
(22) Tokmakoff, A. Time-dependent quantum mechanics and spectroscopy. Lecture, 2014.

(23) Raab, A.; Worth, G. A.; Meyer, H.-D.; Cederbaum, L. S. Molecular dynamics of pyrazine after excitation to the S2 electronic state using a realistic 24-mode model Hamiltonian. J. Chem. Phys. 1999, 110, 936-946.

(24) Vendrell, O.; Meyer, H. D. Multilayer multiconfiguration timedependent Hartree method: Implementation and applications to a Henon-Heiles Hamiltonian and to pyrazine. J. Chem. Phys. 2011, 134, 044135.

(25) Yabana, K.; Bertsch, G. Time-dependent local-density approximation in real time. Phys. Rev. B: Condens. Matter Mater. Phys. 1996, 54, 4484-4487.

(26) De Giovannini, U.; Brunetto, G.; Castro, A.; Walkenhorst, J.; Rubio, A. Simulating pump-probe photoelectron and absorption spectroscopy on the attosecond timescale with time-dependent density functional theory. ChemPhysChem 2013, 14, 1363-1376.

(27) McLachlan, A. D. A variational solution of the time-dependent Schrodinger equation. Mol. Phys. 1964, 8, 39-44.

(28) Vacher, M.; Bearpark, M. J.; Robb, M. A. Direct methods for non-adiabatic dynamics: connecting the single-set variational multiconfiguration Gaussian (vMCG) and Ehrenfest perspectives. Theor. Chem. Acc. 2016, 135, 187.

(29) Li, X.; Tully, J. C.; Schlegel, H. B.; Frisch, M. J. Ab initio Ehrenfest dynamics. J. Chem. Phys. 2005, 123, 084106.

(30) Andrea Rozzi, C.; Maria Falke, S.; Spallanzani, N.; Rubio, A.; Molinari, E.; Brida, D.; Maiuri, M.; Cerullo, G.; Schramm, H.; Christoffers, J. Quantum coherence controls the charge separation in a prototypical artificial light-harvesting system. Nat. Commun. 2013, 4, 1602.

(31) Krumland, J.; Valencia, A. M.; Pittalis, S.; Rozzi, C. A.; Cocchi, C. Understanding real-time time-dependent density-functional theory simulations of ultrafast laser-induced dynamics in organic molecules. J. Chem. Phys. 2020, 153, 054106.

(32) Goings, J. J.; Lingerfelt, D. B.; Li, X. Can quantized vibrational effects be obtained from ehrenfest mixed quantum-classical dynamics? J. Phys. Chem. Lett. 2016, 7, 5193-5197.

(33) Kapral, R.; Ciccotti, G. Mixed quantum-classical dynamics. J. Chem. Phys. 1999, 110, 8919-8929.

(34) Broeckhove, J.; Lathouwers, L.; Kesteloot, E.; Van Leuven, P. On the equivalence of time-dependent variational principles. Chem. Phys. Lett. 1988, 149, 547-550.

(35) Lubich, C. On variational approximations in quantum molecular dynamics. Mathematics of Computation 2005, 74, 765-780.

(36) Ohta, K. Time-dependent variational principle with constraints for parametrized wave functions. Phys. Rev. A: At., Mol., Opt. Phys. 2004, 70, 022503.

(37) Kosloff, R.; Tal-Ezer, H. A direct relaxation method for calculating eigenfunctions and eigenvalues of the schrödinger equation on a grid. Chem. Phys. Lett. 1986, 127, 223.

(38) Shi, T.; Demler, E.; Ignacio Cirac, J. Variational study of fermionic and bosonic systems with non-Gaussian states: Theory and applications. Ann. Phys. 2018, 390, 245-302.

(39) Generalized Inverses; Springer: New York, NY, 2006.

(40) Kreibich, T.; Lein, M.; Engel, V.; Gross, E. K. Even-harmonic generation due to beyond-born-oppenheimer dynamics. Phys. Rev. Lett. 2001, 87, 103901.

(41) Lein, M.; Kreibich, T.; Gross, E. K.; Engel, V. Strong-field ionization dynamics of a model [Formula Presented] molecule. Phys. Rev. A: At., Mol., Opt. Phys. 2002, 65, 033403.

(42) Bandrauk, A. D.; Shon, N. H. Attosecond control of ionization and high-order harmonic generation in molecules. Phys. Rev. A: At., Mol., Opt. Phys. 2002, 66, 031401.

(43) Crespo-Otero, R.; Barbatti, M. Spectrum simulation and decomposition with nuclear ensemble: Formal derivation and application to benzene, furan and 2-phenylfuran. Theor. Chem. Acc. 2012, 131, 1237.

(44) Tancogne-Dejean, N.; Oliveira, M. J.; Andrade, X.; Appel, H.; Borca, C. H.; le Breton, G.; Buchholz, F.; Castro, A.; Corni, S.;
Correa, A. A.; et al. Octopus, a computational framework for exploring light-driven phenomena and quantum dynamics in extended and finite systems. J. Chem. Phys. 2020, 152, 124119.

(45) Gross, E. K. U.; Maitra, N. T. Introduction to TDDFT. Fundamentals of Time-Dependent Density Functional Theory 2012, 837, 53-99.

(46) Pantos, E.; Philis, J.; Bolovinos, A. The extinction coefficient of benzene vapor in the region 4.6 to $36 \mathrm{eV}$. J. Mol. Spectrosc. 1978, 72, $36-43$.

(47) Koch, E. E.; Otto, A. Optical absorption of benzene vapour for photon energies from 6 to $35 \mathrm{eV}$. Chem. Phys. Lett. 1972, 12, 476480.

(48) Gingell, J. M.; Marston, G.; Mason, N. J.; Zhao, H.; Siggel, M. R. On the electronic spectroscopy of benzyl alcohol. Chem. Phys. 1998, 237, 443-449.

(49) Ridolfi, E.; Trevisanutto, P. E.; Pereira, V. M. Expeditious computation of nonlinear optical properties of arbitrary order with native electronic interactions in the time domain. Phys. Rev. B: Condens. Matter Mater. Phys. 2020, 102, 245110.

(50) Verlet, L. Computer "experiments" on classical fluids. I. Thermodynamical properties of Lennard-Jones molecules. Phys. Rev. 1967, 159, 98. 